\title{
AVALIAÇÃO DA PRESSÃO ARTERIAL EM HIPERTENSOS PRATICANTES DE ATIVIDADE FÍSICA
}

\section{PRESSURE ASSESSMENT IN BLOOD HYPERTENSIVE PHYSICAL ACTIVITY PRACTITIONERS}

\author{
Elenice Gomes Ferreira*, Patrícia Almeida Pereira**, Andiárie Nogueira Costa**, Mateus Dias \\ Antunes***, Daniel Vicentini de Oliveira****
}

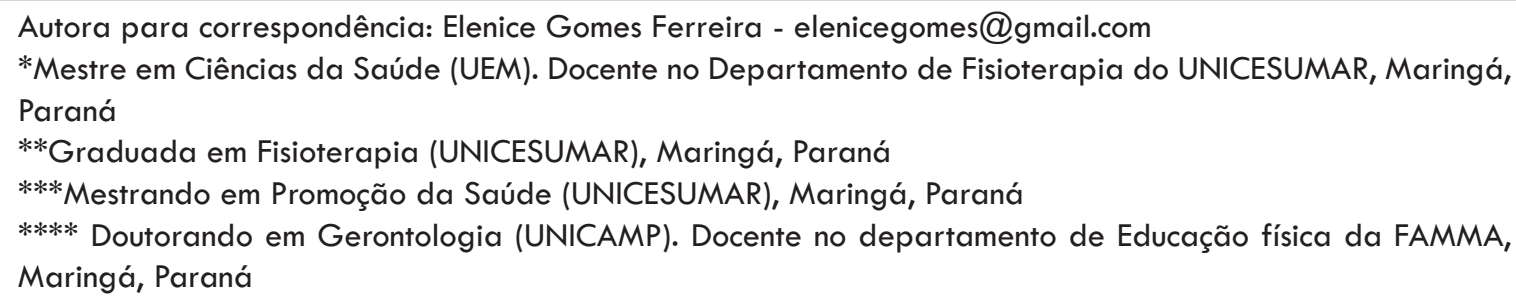

R E S U M O

\begin{abstract}
Objetivo: Avaliar a alteração da pressão arterial em indivíduos hipertensos usuários de uma das Academias da Terceira Idade em dois horários diferentes do dia. Metodologia: Trata-se de um estudo observacional de corte transversal. A amostra foi composta por 40 indivíduos de ambos os sexos, hipertensos, e que faziam o uso de medicamentos anti-hipertensivos, com idade igual ou superior a 50 anos, usuários das Academias da Terceira Idade do município de Maringá, estado do Paraná. Estatura, peso, índice de massa corporal, circunferência abdominal, aferição da pressão arterial e avaliação da qualidade de vida por meio do Minichal-Brasil, foram as variáveis analisadas. Baseado em estatística descritiva por meio de números absolutos e relativos, média e desvio padrão os dados foram apresentados. Resultados: Houve predomínio do sexo masculino e a média de idade foi de 60,55 anos. Ambos os sexos apresentaram sobrepeso, sendo que as mulheres obtiveram maiores índices de circunferência abdominal. Foram semelhantes os níveis pressóricos sistólicos estando entre os parâmetros de normalidade, já os diastólicos após a realização do exercício físico quando comparados com as mensurações iniciais, não houve grandes alterações em ambos os períodos de aferição diária. Conclusão: Conclui-se que não houve grandes alterações da pressão arterial sistólica e diastólica nas diferentes faixas etárias de acordo com o turno da manhã e da noite de forma a expor os indivíduos a ataques cardiovasculares. Indivíduos hipertensos, praticantes de atividades físicas e que fazem o uso regular de medicamento anti-hipertensivo, mostraram manter uma boa qualidade de vida.
\end{abstract}

Palavras-chave: Atividade física; Doença cardiovascular; Hipertensão. 
Objective: To evaluate the change in blood pressure in hypertensive individuals users of thewho attend gyms for seniors at two different times of the day. Metodology: This is an observational and crosssectional study. The sample consisted of 40 subjects of both genders, hypertensive, and that made the use of antihypertensive medications, aged over 50 years, users of the Academies of the Third Age of the city of Maringa, Parana State. Height, weight, body mass index, waist circumference, blood pressure measurement and evaluation of quality of life for Minichal-Brasil, were the variables analyzed. Based on descriptive statistics through absolute and relative mean and standard deviation data were presented. Results: There was a predominance of males and the average age was 60.55 years. Both sexes were overweight, and women had higher rates of abdominal circumference. They were similar to the systolic levels being among the normal parameters, since diastolic after the completion of the exercise when compared with the initial measurements, there were no major changes in both periods of daily verification. Conclusion: It was concluded that there were no major changes in systolic and diastolic blood pressure in different age groups according to the morning shift and night in order to expose individuals to cardiovascular attacks. Individuals hypertension, physical activity and practitioners who make regular use of antihypertensive medication, showed maintain a good quality of life.

Keywords: Physical activity; Cardiovascular disease; Hypertension. 


\section{INTRODUÇÃO}

O Brasil tem apresentado um acelerado processo de envelhecimento com grandes transformações na composição etária e epidemiológica da sua população'. Atualmente com a melhora da educação em relação à saúde e da estrutura do sistema de saúde ${ }^{2}$, habitação e saneamento das cidades, ocorreu uma expectativa e qualidade de vida maior, papéis importantíssimos na longevidade da vida humana ${ }^{3}$.

A hipertensão arterial sistêmica (HAS) é considerada um dos maiores problemas de saúde pública, tendo alta prevalência e baixo controle, se não for tratada é um importante fator de risco para doenças cardiovasculares ${ }^{4}$. Atualmente ocorrem grandes alterações comportamentais na sociedade que incentivam o sedentarismo e o consumo de bebidas e alimentos com alto teor calórico ${ }^{5}$. Para prevenir ou tratar a hipertensão, assim como outras doenças cardiovasculares, é necessário a modificação de diversos hábitos inadequados de saúde de toda a populaçãob.

Os benefícios da atividade física no tratamento e prevenção da HAS faz com que ocorra o controle do peso corporal e melhora da mobilidade articular e da força muscular, bem como da resistência física. O aumento da densidade óssea, maior resistência à insulina e redução da pressão arterial também são efeitos que a prática de atividade física proporciona ${ }^{7}$. Para que o tratamento não medicamentoso tenha sucesso, é necessário o acompanhamento de um profissional da saúde habilitado, com o propósito de alcançar o melhor resultado por meio de uma abordagem multidisciplinar e interdisciplinar ${ }^{8}$.

Aproximadamente 90 a $95 \%$ dos casos de HAS são primárias e de 5 a $10 \%$ são secundárias por causa renal, endócrina, cardiovascular e neurológica?. A HAS é influenciada por fatores ambientais como a ingesta de sódio, sensibilidade ao sal, idade, climatério, obesidade, resistência à insulina, sedentarismo, tabagismo, alcoolismo e estresse, também pode ser causada por fatores genéticos devido à etnia e gênero ${ }^{10}$.

Contudo a prevalência de HAS no Brasil, tem sido analisada através de inquéritos populacionais nas cidades nos últimos anos, mostrando que pelo menos $30 \%$ da população adulta tem pressão arterial igual ou superior a $140 / 90 \mathrm{mmHg}$, sendo que $50 \%$ estão com idade entre 60 e 69 anos e $75 \%$ acima dos 70 anos, e nos gêneros a incidência é de $35,8 \%$ nos homens e $30 \%$ nas mulheres ${ }^{10}$.

Uma sessão exagerada de exercício pode aumentar o risco de um evento adverso clínico, podendo ocorrer a morte súbita cardíaca ou infarto do miocárdio, principalmente em indivíduos vulneráveis ${ }^{11-12}$. Uma vez que já foi descoberto que a incidência desses eventos tem picos durante a manhã e que alguns pacientes cardíacos preferem agendar $\circ$ lazer e atividade física neste horário, a questão é saber se o exercício da manhã se torna mais arriscado do que a atividade física realizada em outros horários do dia ${ }^{13}$. Entretanto, poucos estudos têm sido desenvolvidos a fim de examinar se tais variáveis de pressão arterial respondem diferentemente o nível de exercício no período da manhã em comparação com o período do fim da tarde ${ }^{14}$.

Este estudo teve como objetivo avaliar a alteração da pressão arterial em indivíduos hipertensos usuários da academia das academias da terceira idade em dois horários diferentes do dia.

\section{MATERIAL E MÉTODOS}

Este estudo caracterizou-se como quantitativo e transversal realizado no período de junho a setembro de 2013, aprovado pelo Comitê de Ética em Pesquisa em Seres Humanos do UNICESUMAR por meio do parecer $\mathrm{n}^{\circ}$ 255.880/2013 (CAAE 13715213.5.0000.5539).

Foram selecionados 60 indivíduos de ambos os sexos, com HAS e que fazem uso de medicamentos anti-hipertensivos, com idade igual ou superior a 50 anos, usuários das Academias da Terceira Idade (ATI) do município de Maringá, estado do Paraná, e que concordaram em participar do estudo após assinarem o Termo de Consentimento Livre e Esclarecido (TCLE) que foi preenchido em duas vias, 
permanecendo uma em posse dos pesquisadores e outra em posse do indivíduo pesquisado.

Para os critérios de exclusão foram considerados os participantes com diagnóstico clínico de insuficiência cardíaca, acidente vascular encefálico, doença isquêmica miocárdica, insuficiência renal crônica, individuo que inadequadamente faziam $\circ$ uso de anti-hipertensivos, e não comparecimentos no próximo dia de avaliação.

A avaliação dos indivíduos foi realizada em dois dias consecutivos, sendo que no primeiro dia, no período da noite, e o segundo no início da manhã pois são turnos que maior expões a ataques cardíacos. Todos os indivíduos pesquisados foram submetidos a uma avaliação de aferição da pressão arterial (PA), já tendo feito a administração do medicamento antihipertensivo, teste de qualidade de vida, medida de circunferência abdominal (CA), estatura, peso, e Índice de Massa Corporal (IMC). Após a avaliação, - programa de exercícios foi elaborado pelo próprio participante de forma que cada avaliado realizasse os exercícios durante meia hora sem nenhuma intervenção dos pesquisadores. Ao término do mesmo, foi feita uma nova aferição da PA. No segundo dia de avaliação, foi feito a aferição da PA antes do indivíduo realizar a atividade física, e o mesmo deveria realizar os exercícios novamente sem a intervenção dos pesquisadores durante no mínimo meia hora, e após foi aferida sua PA novamente.

Para mensurar a pressão arterial sistólica e diastólica dos indivíduos, os pesquisadores localizaram a artéria braquial ao longo da face interna superior do braço, usando esfigmomanômetros mecânicos aneróides, em conjunto com estetoscópios, ambos da marca Premium. Na primeira aferição o paciente permaneceu sentado por 5 minutos, com $\circ$ braço apoiado ao nível do coração, e a segunda aferição foi realizada com o mesmo posicionamento sem $\circ$ tempo de 5 minutos, logo após o fim do exercício.

O peso corporal foi verificado por meio de uma balança da marca Digital EcoLine 180. Para a cirtometria abdominal, foi utilizada a cicatriz umbilical como ponto de referência o indivíduo foi posicionado de forma ereta, com o olhar para o horizonte e braços ao longo do corpo. A estatura foi verificada através de uma fita métrica da marca Cirúrgica Express, enquanto o IMC foi calculado pela divisão do peso corporal $(\mathrm{kg})$, pelo quadrado da estatura (metros).

A avaliação da qualidade de vida foi através do teste Minichal-Brasil, sendo um instrumento específico e validado para indivíduos com HAS, de fácil administração e boa confiabilidade. O questionário contém 17 questões de múltipla escolha separadas em dois fatores: estado mental, contendo 10 questões, manifestações somáticas, contendo 6 questões, e a última questão o indivíduo avalia como o tratamento da HAS teve influência na sua qualidade de vida, sendo que os participantes deveriam responder as questões considerando seus últimos sete dias. As respostas foram assinaladas através da escala de frequência do tipo Likert, tendo quatro opções de respostas, sendo 0 (não, absolutamente), 1 (sim, um pouco), 2 (sim, bastante) e 3 (sim, muito). A pontuação máxima para as questões relacionadas ao estado mental é de 30 pontos e para as manifestações somáticas 18 pontos, quanto mais próximo ao 0 for 0 resultado, melhor a qualidade de vida do mesmo ${ }^{15}$.

A análise dos dados foi realizada por meio da estatística descritiva (números absolutos e relativos, média e desvio padrão).

\section{RESULTADOS}

O período da coleta de dados foi de junho a setembro de 2013, onde inicialmente foram selecionados 60 indivíduos, porém, 20 desistiram de participar do estudo. Dentre os 40 participantes, $67 \%(n=27)$ eram do sexo masculino.

Os participantes apresentavam faixa etária entre 50 e 90 anos, sendo a média de idade de 60,55 anos. Foram alocados em dois grupos sendo 0 Grupo 1 de 50 a 70 anos contendo 12 mulheres e 25 homens, e Grupo 2 de 71 a 90 anos contendo 1 mulher e 2 homens.

No presente estudo, pode-se observar que a média em relação ao IMC dos homens e das mulheres da amostra foi igual, representando sobrepeso, e as mulheres apresentaram-se com a média de CA 
acima do estimado quando comparado aos homens. Por meio da análise da Tabela 1, verifica-se em ambas as faixas etárias que a pressão arterial sistólica inicial (PASi) tanto no período da manhã quanto da noite, apresentaram-se semelhantes $e$ em níveis adequados. Quanto ao valor da pressão arterial sistólica após o exercício físico, comparada à aferição inicial, nota-se em seu comportamento que não houve grandes alterações nos dois períodos do dia. Contudo, o grupo de 71-90 anos após a prática de exercício físico no período da noite, apresentou um aumento de $17 \mathrm{mmHg}$, sendo 7 $\mathrm{mmHg}$ em relação a PASi e PASf da manhã.

Tabela 1. Média de pressão arterial sistólica inicial e final segundo faixa etária em turnos distintos.

\begin{tabular}{lllll}
\hline Faixa etária & \multicolumn{2}{c}{ PASi (D.P) } & \multicolumn{2}{c}{ PASf (D.P) } \\
\cline { 2 - 5 } & Manhã & Noite & Manhã & Noite \\
\hline Grupo 1 & $129( \pm 36)$ & $130( \pm 36)$ & $141( \pm 36)$ & $140( \pm 36)$ \\
Grupo 2 & $133( \pm 2)$ & $133( \pm 2)$ & $143( \pm 2)$ & $150( \pm 2)$ \\
\hline
\end{tabular}

*PASi: Pressão arterial sistólica inicial; **PASf: Pressão arterial sistólica final). D.P: desvio padrão

$\mathrm{Na}$ Tabela 2 a seguir observa-se que $\circ$ comportamento da pressão arterial diastólica (PAD) antes e após o exercício no grupo de 50-70 anos apresentou-se semelhante nos diferentes períodos, tendo uma variação de $3,7 \mathrm{mmHg}$ e $4,4 \mathrm{mmHg}$ respectivamente, enquanto que no grupo de 71-90 anos houve um aumento no período da noite, sendo que pela manhã não houve alteração.

Tabela 2. Média de pressão arterial diastólica inicial e final segundo faixa etária em turnos distintos.

\begin{tabular}{lllll}
\hline Faixa etória & \multicolumn{2}{c}{ PASi (D.P) } & \multicolumn{2}{c}{ PASf (D.P) } \\
\cline { 2 - 5 } & \multicolumn{1}{c}{ Manhã } & Noite & Manhã & Noite \\
\hline Grupo 1 & $81.3( \pm 36)$ & $81.6( \pm 36)$ & $85( \pm 36)$ & $86( \pm 36)$ \\
Grupo 2 & $86( \pm 2)$ & $93( \pm 2)$ & $86( \pm 2)$ & $100( \pm 2)$ \\
\hline
\end{tabular}

*PADi: Pressão arterial diastólica inicial; **PADf: Pressão arterial diastólica final.

Em relação à avaliação do questionário de qualidade de vida MINICHAL-BRASIL, pode ser observado que através da média obtida das questões, de acordo com a escala de Likert, que as questões que abrangiam o estado mental, foram as mais assinaladas negativamente (média 6,35).

\section{DISCUSSÃO}

No presente estudo, além dos pacientes serem hipertensos, verificou a presença de outros fatores de risco para doenças cardiovasculares, como o IMC foi representado com sobrepeso em ambos os gêneros e CA elevada apenas nas mulheres. Foi visto também que o grupo de maior faixa etária, apresentava maiores alterações de pressão arterial. Um dos principais fatores de risco associado à hipertensão é o excesso de gordura corporal, identificada pelo IMC, e a gordura abdominal pela medida da $\mathrm{CA}^{16}$ Estes mesmos autores ${ }^{17}$ também apresentaram resultados que mostravam que a 
presença de hipertensão arterial tem associação com IMC elevado em ambos os sexos.

Com o resultado do presente estudo, foi possível observar que não houve grandes alterações de PAS e PAD após exercício de acordo com diferentes períodos do dia (ritmo biológico), pois as médias dos resultados permaneceram estáveis e dentro do padrão de normalidade para indivíduos hipertensos que se medicam, afastando-os de manifestações de riscos, opondo com outro estudo ${ }^{17}$ em que as primeiras horas do dia são as mais perigosas tratando-se de ataques cardiovasculares, com pico entre 06:00 e 12:00 horas da manhã, de acordo com o ritmo biológico e as alterações hemodinâmicas e funcionais que ocorrem no corpo durante esses horários.

Em outro estudo ${ }^{14}$ descreve que por meio do ritmo biológico pode-se ter uma menor variação de pressão arterial através de exercícios realizados a noite. Porém, deve-se levar em conta a relação de outros fatores que propiciam a mortalidade por doenças cardiovasculares em horários de menor pico como estressem sedentarismo, alimentação, consumo de álcool e medicação inadequada. No presente estudo o grupo 71-90 anos, ao realizar o exercício à noite, obteve um aumento considerável na PAS e PAD após seu término, podendo ser este aumento devido a esses fatores.

Foi verificado no presente estudo que a variação da PA após exercício foi pequena durante a manhã em relação ao aumento no período da noite, o que difere dos resultados de outro estudo ${ }^{18}$, os quais resultaram em maior elevação da pressão arterial sistólica e diastólica no período da manhã.

Observa-se que de acordo com as médias de PASf e PADf tanto no período da manhã quanto da noite, houve de fato uma elevação de pressão arterial pós exercício físico, em relação a pressão arterial mensurada no início, porém sem picos hipertensivos, semelhante em outro estudo ${ }^{19}$, em que o comportamento da pressão arterial pré e pós exercício físico, nos mostrando que a pressão arterial sistólica e diastólica aumentam após a atividade física.

Por meio do questionário Minichal-Brasil, observa-se pelas médias que os indivíduos apresentaram boa qualidade de vida de acordo com suas próprias opiniões, tanto no fator de estado mental quanto no de manifestação somática, tendo o resultado mais próximo a zero (boa qualidade de vida) do que de 30 (má qualidade de vida). Uma possível explicação para este resultado pode ser devido à seleção de indivíduos, que eram praticantes de atividade física e faziam o uso de medicamento anti-hipertensivo regularmente, se assemelhando com o estudo ${ }^{26}$ que compara indivíduos que tiveram bons resultados no escore por serem mais informados e tomarem devidas precauções quanto à doença.

Algumas limitações importantes do estudo devem ser discutidas, como o tamanho amostral reduzido e o número de estudos que comparam a pressão arterial no período da manhã e da noite ainda são escassos, porém, pesquisadores afirmam que de fato a elevação da pressão sistólica e diastólica no período da manhã é maior que no período noturno.

\section{CONCLUSÃO}

Conclui-se que não houve grandes alterações da pressão arterial sistólica e diastólica nas diferentes faixas etárias de acordo com o turno da manhã e da noite de forma a expor os indivíduos a ataques cardíacos e que os indivíduos que são hipertensos, praticantes de atividades físicas e que fazem o uso regular de medicamento anti-hipertensivo apresentam uma manutenção da qualidade de vida. Sugerem-se novos estudos a fim de comprovar os resultados para que possam promover ações de promoção e prevenção da saúde.

\section{REFERÊNCIAS}

1. Mari FR, Alves GG, Aerts DRGC, Camara S. O processo de envelhecimento e a saúde: o que pensam as pessoas de meia-idade sobre o tema. Rev Bras Geriatr Gerontol. 2016;19(1):35-44. doi: $10.1590 / 1809-9823.2016 .14122$ 
2. Pinto $A M$, Rodrigues $V$, Botelho $A$, Veríssimo $M T$, Morais A, Rosa MS et al. A socio-demographic study of aging in the Portuguese population: The EPEPP study. Arch Gerontol Geriatr. 2011 ;52(3):304-8. doi: 10.1016/2010.04.019

3. Fechine BRA, Trompieri N. O processo de envelhecimento: as principais alterações que acontecem com o idoso com o passar dos anos. Inter Science Place. 2015; (20):106-32. doi: $10.6020 / 1679-9844 / 2007$

4. Yan $Q$, Sun $D$, Li X, Chen $G$, Zheng $Q$, Li L et al. Association of blood glucose level and hypertension in Elderly Chinese Subjects: a community based study. BMC Endocr Disord. 2016;16(1):1-8. doi: 10.1186/s12902-016-0119-5

5. Dutra DD, Duarte MCS, Albuquerque KF, Lima AS, Santos JS, Souto HC. Cardiovascular disease and associated factors in adults and elderly registered in a basic health unit. Rev Pesq Cuid Fund. J Res Care Essen. 2016;8(2):4501-4509. doi: 10.9789/2175-5361.2016.v8i2.4501-4509

6. Dias EG, Souza ELS, Mishima SM. Contribuições da Enfermagem na adesão ao tratamento da hipertensão arterial: uma revisão integrativa da literatura brasileira. Rev Epidemiol Cont Infec. 2016;6(3):45-39. doi: 10.17058/reci. v6i3.7470

7. Yazawa A, Inoue Y, Fujiwara T, Stickley A, Shirai K, Amemiya A et al. Association between social participation and hypertension among older people in Japan: the JAGES Study. Hypertens Res. 2016;39(11):818-824. doi: 10.1038/hr.2016.78

8. Yang SO, Kim SJ, Lee Sh. Effects of a South Korean Community-Based Cardiovascular Disease Prevention Program for LowIncome Elderly with Hypertension. J Commun Heath Nurs. 2016;33(3):154-67. doi: $10.1080 / 07370016.2016 .1191872$

9. Rodrigues C, Silva JP, Cabral CVS. Fatores de risco para o desenvolvimento de hipertensão arterial (HAS) entre a equipe de enfermagem. Rev Interd. 2016;9(2):1 17-26. doi: 10.5205/ reuol.9373-82134-1-RV1008201646
10. Nunes T, Martins A, Manoel A, Trevisol $D$, Schuelter-Trevisol F, Cavalcante $R$ et al. Hypertension in elderly individuals from a city of Santa Catarina: a population-based study. Int J Cardio Scienc. 2015;28(5):370-376. doi: $10.5935 / 2359-4802.20150055$

11. Rosário TM, Scala LCN, França GVA, Pereira $M R G$, Jardim PCBV. Prevalence, control and treatment of arterial hypertension in NobresMT. Arq. Bras. Cardiol. 2009;93(6):672-78. doi: $10.1590 /$ S0066-782X2009001200018

12. Vitorino GFA, Oliveira MI, Araúio HVS, Belo RMO, Figueirêdo TR, Bezerra SMMS. Perfil de saúde e qualidade de vida de idosas com hipertensão arterial sistêmica. Rev Rene. 2015;16(6):900-7. doi: 10.15253/21756783.2015000600017

13. Zamai CA, Peres CM, Bonfin ES, Souza JP, Leme PAF, Guerra RLS. Intervenção de atividade física na hipertensão arterial e diabetes de servidores da UNICAMP. SMTEC. 2016;5(1):156-67. doi: $10.20396 /$ sinteses.v0i5.7189

14. Silva RLDT, Barreto MDS, Arruda GOD, Marcon SS. Evaluation of the care program implementation to people with high blood pressure. Braz J Nurs. 2016;69(1):79-87. doi: 10.1590/0034$7167.2016690111 \mathrm{i}$

15. Schulz RB, Rossignoli P, Correr CJ, FernándezLlimós F, Toni PM. Validação do mini-questionário de qualidade de vida em hipertensão arterial (MINICHAL) para o português (Brasil). Arq Bras Cardiol. 2008;90(2):139-44. doi: 10.1590/ S0066-782X2008000200010

\section{Zattar LC, Boing AF, Giehl MWC, D'Órsi} E. Prevalência e fatores associados à pressão arterial elevada, seu conhecimento e tratamento em idosos no sul do Brasil. Cad Saúde Pública. 2013;29(3):507-21. doi: 10.1590/S0102$311 \times 2013000300009$

17. Walther D, Curjuric I, Dratva J, Schaffner E, Quinto C, Rochat T, et al. High blood pressure: prevalence and adherence to guidelines in a population-based cohort. Swiss Med Wkly. 2016;146(2):143-153. doi: 10.4414/ 
smw.2016.14323

18. Silva GCA, Pierin AMG. A monitorização residencial da pressão arterial e o controle de um grupo de hipertensos. Rev Esc Enferm USP. 2012;46(4):922-28. doi: 10.1590/S008062342012000400020

19. Liao Y, Siegel PZ, White S, Dulin R, Taylor A. Improving actions to control high blood pressure in Hispanic communities-Racial and Ethnic Approaches to Community Health Across the US Project, 2009-2012. Prevent Med. 2016:83(1):11 15. doi: 10.1016/i.ypmed.2015.11.027 